\title{
Comparative and Computer Assisted Drug Designing of Fatty Acids Isolated from Flowers, Leaves, Stem Bark, Root Bark and Nuts of Semecarpus anacardium L. $f$. (Anacardiaceae)
}

Vustelamuri Padmavathi ${ }^{1}$, Bhattiprolu Kesava Rao ${ }^{1}$, Noboru Motohashi ${ }^{2}$, Sridhara Janardhan ${ }^{3}$ and G. Narahari Sastry $^{3}$

1. Department of Chemistry, University College of Sciences, Acharya Nagarjuna University, Nagarjunanagar 522510, Guntur District, Andhra Pradesh, India

2. Meiji Pharmaceutical University, 2-522-1 Noshio, Kiyose-shi 204-8588, Tokyo, Japan

3. Centre for Molecular Modeling, CSIR-Indian Institute of Chemical Technology, Tarnaka, Hyderabad 500007, India

\begin{abstract}
Objectives: Computational study will help us in reducing the experimental work. The process of drug discovery involves the designing of molecules with appropriate pharmacophores with the help of various soft wares. The purpose of this paper is to study the probable binding modes of fatty acids on fatty acids after enzymatic hydrolysis of the FAAH (fatty acid amide hydrolase) in different extracts of flowers, leaves, stem bark, root bark and nuts of Semecarpus anacardium L. f. by using molecular modeling study and computer assisted drug designing. Nuts yielded 20 fatty acids including saturated, $\omega-3$ unsaturated, $\omega-6$ unsaturated, $\omega-7$ unsaturated and $\omega^{-9}$ unsaturated fatty acids. Based on IR, ${ }^{1} \mathrm{H}$ NMR, ${ }^{13} \mathrm{C}$ NMR, MS (mass) spectrometry, GC analysis, the structural elucidation of these isolated fatty acids was established. Methods: A dataset comprising of 20 fatty acids were drawn in ChemDraw and converted into 3D-molecules with all possible tautomers and chiral centers. The minimization of molecules was carried out using PRCG (Polak-Ribiere Conjugate Gradient) method with maximum of 5000 iterations. The minimized compounds were used for protein preparation. The crystal structure of human FAAH (PDB ID: 3K84) is prepared and selected for the docking studies of 20 fatty acids using Schrödinger docking program module.. Conclusions: In this study, we carried out the molecular docking studies in order to understand the probable binding mode of 20 fatty acids in FAAH from which we identified key active site residues for FAAH, thereby it can be used to design the novel compounds for FAAH targets.
\end{abstract}

Key words: Computer assisted, drug designing, fatty acids, Semecarpus anacardium L. f., $\omega$-unsaturated fatty acids.

\section{Introduction}

Semecarpus anacardium L. f. [1] (Anacardiaceae) is reported to possess many medicinal properties. Trees will grow up to $25 \mathrm{~m}$ tall, young branches are terete and tomentose, and watery latex is present, which on drying turns to black. The black corrosive juice of the pericarp contains tarry oil [2] consisting of $90 \%$ of oxy-acid, anacardic acid and $10 \%$ of higher

Corresponding author: Bhattiprolu Kesava Rao, Ph.D., professor, research field: organic chemistry-natural products and medicinal chemistry. E-mail: krbhattiprolu@gmail.com. nonvolatile alcohol called cardol, also contains catechol and a mono-hydroxy phenol called as anacardol. The most significant components of Semecarpus anacardium L. f. oil are phenolic compounds. On exposure to air, phenolic compounds get oxidized to quinones. The oxidation process can be prevented by keeping the oil under nitrogen.

\section{Materials and Methods}

All the melting points were recorded in a Toshniwal melting point apparatus. FT-IR spectra of the fatty 
acids were recorded using the $\mathrm{KBr}$ pellet method on Perkin-Elmer Spectrum 400 FT-IR, FT-NMR-The spectrum ${ }^{\mathrm{TM}} 400 .{ }^{1} \mathrm{H}$ NMR and ${ }^{13} \mathrm{C}$ NMR spectra of the fatty acids were taken on Bruker $300 \mathrm{MHz}$ NMR Spectrometer using $\mathrm{CDCl}_{3}$ as a solvent. Mass spectra were recorded on LC-MS/GCMS. TLC was carried out using silica-gel (Merk, 100-200 mesh).

\subsection{Plant Material}

Semecarpus anacardium L. f. nuts were collected from field area very nearer to the village Nandgaon, the area covering 20-25 km outskirts of Kolhapur City, Maharashtra, India. All plant material specimens were identified by Dr. Vatsavaya S. Raju, M.Sc., Ph.D., D.A.S., FBS, FIAT retired professor, former head and chairperson, BOS Plant Systematic Laboratory, Department of Botany, Kakatiya University, Warangal (AP) and confirmed as Semecarpus anacardium L. f. (syn: Anacardium latifolium Lam., A. orientale Steud.) of Anacardiaceae and plant specimen deposited at Kakatiya University Herbarium, Warangal (KUW) with accession number 1,874 . It is locally known as "nalla Jeedi" and popularly known as "marking nut/dhobi nut".

\subsection{Extraction and Purification}

Nuts: $3 \mathrm{~kg}$ nuts of plant material Semecarpus anacardium L. f. were collected and dried in the shade. The nuts were directly percolated with cold petroleum ether 5-6 times, filtered and concentrated under vacuum distillation.

Step 1: The nuts were made into small pieces, dried and percolated with petroleum ether 5-6 times, filtered and concentrated under vacuum distillation;

Step 2: After that, the powdered nuts were percolated with hot petroleum ether 5-6 times, filtered and concentrated under vacuum distillation;

Step 3: Later, the same powdered nuts were extracted with cold acetone by changing the solvent for every 3 hours in three intervals, filtered and concentrated under vacuum distillation;
Step 4: After cold acetone percolation, nuts were subjected to hot acetone extract. The crude extract was concentrated under vacuum distillation. These were thoroughly analyzed and investigated in our laboratory. From the above 4 steps, 20 fatty acids were isolated and purified for the first time. In order to prove its suitability, first we have examined its seeds and oil. According to our recent results, nuts of Semecarpus anacardium $\mathrm{L}$. $\mathrm{f}$. were found to have 20 fatty acids (Fig. 1).

\subsection{Leaves, Flowers, Stem Bark and Root Bark}

$7 \mathrm{~kg}$ Leaves, $3 \mathrm{~kg}$ flowers, $3 \mathrm{~kg}$ stem bark and $10 \mathrm{~kg}$ root bark were collected individually and dried under shade. After the shade drying, each one of them was powdered and finally got $2 \mathrm{~kg}$ of leaves powder, $1.5 \mathrm{~kg}$ of flowers powder, $2 \mathrm{~kg}$ of stem bark powder and $3 \mathrm{~kg}$ of root bark powder were obtained. These powders were extracted with $3 \mathrm{~L}$ of hexane/methanol by Soxhlet extraction method for $72 \mathrm{~h}$. The excess of solvent from crude extracts was distilled off and the crude was weighed. The individual weight of the extracts was subjected for column chromatography. The individual fractions were purified by crystallization. The total number of fatty acids isolated from Semecarpus anacardium L. f. in our laboratory are as follows: root bark: 8 ; leaves: 3 ; stem bark: 4 and flowers: 3 , and were compared with the total fatty acids (20) present in the nuts as in Table 1. The structures of these isolated fatty acids were shown in Fig. 2.

\subsection{Docking Studies of Various Binding Modes of Fatty} Acids, FAAH Using Molecular Modeling

In the amidase family of protein, FAAH 1 (human fatty acid amide hydrolase) exists as dimer containing PA (palmitic acid), SA (stearic acid) and LA (linoleic acid). FAAH is located in endomembrane system; single-pass membrane protein, and also in cytoplasm. FAAH is highly present in the brain, small intestine, pancreas, skeletal muscle and testis. FAAH is also locked in the kidney, liver, lung, placenta and prostate. 


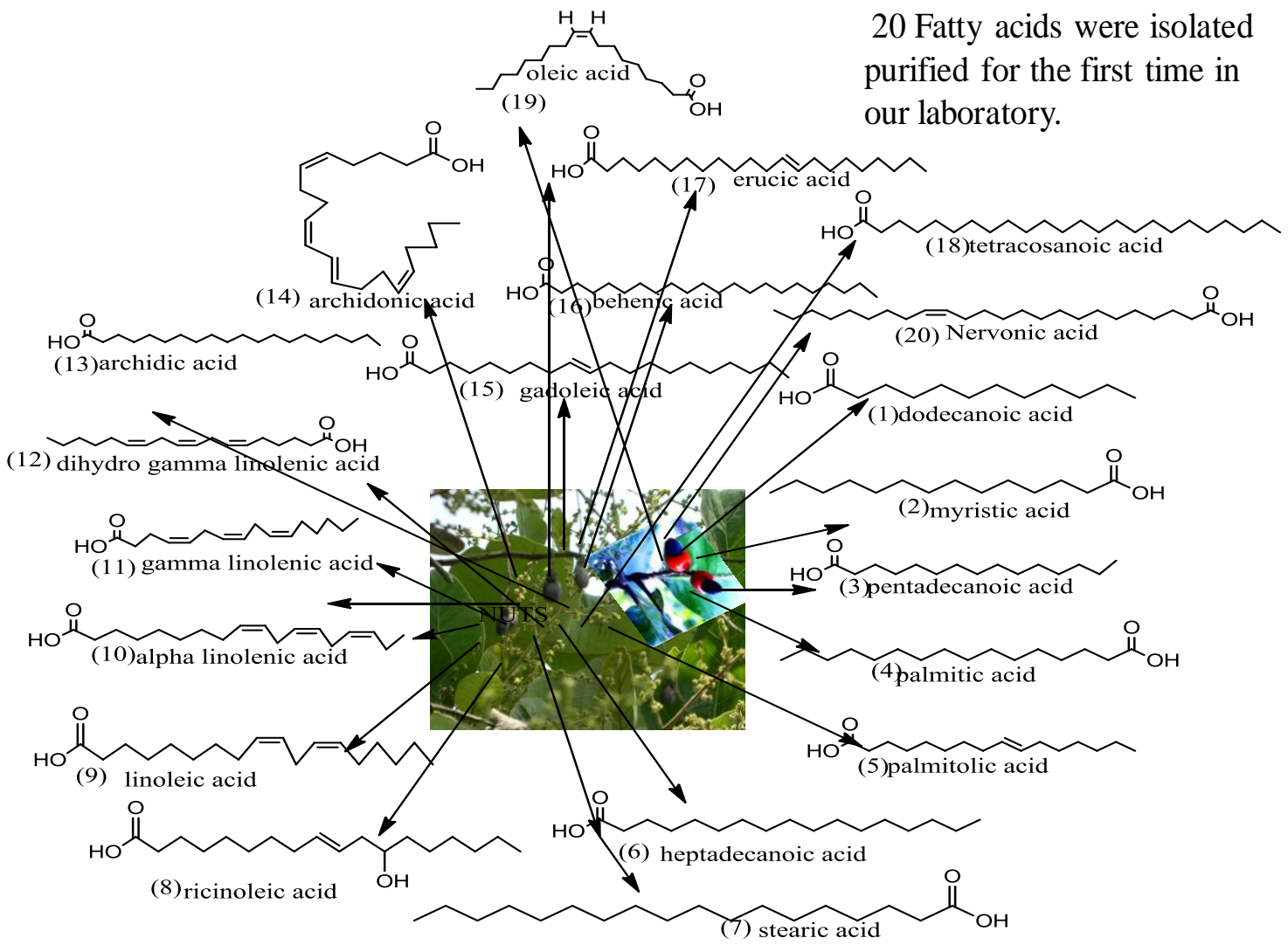

Fig. 1 Fatty acids present in nuts of Semecarpus anacardium L. f..

Table 1 Comparative study of fatty acids present in flowers, leaves, stem barks, root bark and nuts of Semecarpus anacardium L. f..

\begin{tabular}{|c|c|c|c|c|c|c|}
\hline S.NO & Name of the fatty acid & Flowers & Leaves & Stem bark & Nuts & Root bark \\
\hline 1 & LA (lauric acid) & - & - & - & $\sqrt{ }$ & - \\
\hline 2 & MA (myristic acid) & - & - & - & $\sqrt{ }$ & - \\
\hline 3 & PDA (pentadecanoic acid) & & & & $\sqrt{ }$ & \\
\hline 4 & PL (palmitic acid) & $\sqrt{ }$ & $\sqrt{ }$ & $\sqrt{ }$ & $\sqrt{ }$ & $\sqrt{ }$ \\
\hline 5 & PALA (palmitoleic acid) & - & - & - & $\sqrt{ }$ & - \\
\hline 6 & HDA (heptadecanoic acid) & - & - & - & $\sqrt{ }$ & - \\
\hline 7 & SA (stearic acid) & $\sqrt{ }$ & $\sqrt{ }$ & $\sqrt{ }$ & $\sqrt{ }$ & $\sqrt{ }$ \\
\hline 8 & RA (ricinoleic acid) & - & - & - & $\sqrt{ }$ & - \\
\hline 9 & LA (linoleic acid) & - & - & $\sqrt{ }$ & $\sqrt{ }$ & $\sqrt{ }$ \\
\hline 10 & ALA ( $\alpha$-linoleic acid) & - & - & - & $\sqrt{ }$ & - \\
\hline 11 & GLA ( $\gamma$-linolenic acid) & - & - & - & $\sqrt{ }$ & - \\
\hline 12 & DGL (dihydro gamma linolenic acid) & - & - & - & $\sqrt{ }$ & - \\
\hline 13 & AA (archidic acid) & - & - & - & $\sqrt{ }$ & $\sqrt{ }$ \\
\hline 14 & ADA (archidonic acid) & - & - & - & $\sqrt{ }$ & - \\
\hline 15 & GA (gadoleic acid) & - & - & - & $\sqrt{ }$ & - \\
\hline 16 & BA (behenic acid) & $\sqrt{ }$ & - & - & $\sqrt{ }$ & $\sqrt{ }$ \\
\hline 17 & EA (erucic acid) & - & - & - & $\sqrt{ }$ & - \\
\hline 18 & TCA (tetracosanoic acid) & $\sqrt{ }$ & - & $\sqrt{ }$ & $\sqrt{ }$ & $\sqrt{ }$ \\
\hline 19 & OA (oleic acid) & $\sqrt{ }$ & $\sqrt{ }$ & $\sqrt{ }$ & $\sqrt{ }$ & $\sqrt{ }$ \\
\hline 20 & NA (nervonic acid) & - & - & - & $\sqrt{ }$ & $\sqrt{ }$ \\
\hline
\end{tabular}

$\sqrt{ }$ : Present; -: Absent. 
<smiles>CCCCCCCCCCCC(=O)O</smiles>

(1) Lauric acid<smiles>CCCCCCCCCCCCCCC(=O)O</smiles>

(3) Pentadecanoic acid<smiles>CCCCCC/C=C/CCCCCCCC(=O)O</smiles>

(5) Palmitoleic acid<smiles>CCCCCCCCCCCCCCCCCC(=O)O</smiles>

(7) Stearic acid<smiles>CCCCC/C=C\C/C=C\CCCCCCCC(=O)O</smiles>

(9) Linoleic acid<smiles>CCCCC/C=C\C/C=C\C/C=C\CCC(=O)O</smiles>

(11) gamma-Linolenic acid<smiles>CCCCCCCCCCCCCCCCCCCC(=O)O</smiles>

Arachidic acid<smiles>CCCCCCCCCC/C=C/CCCCCCCC(=O)O</smiles>

(15) Gadoleic acid

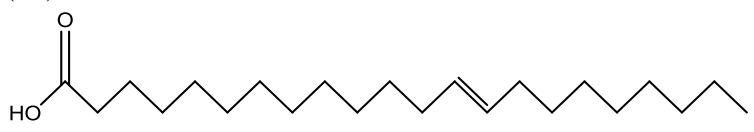

(17) Erucic acid

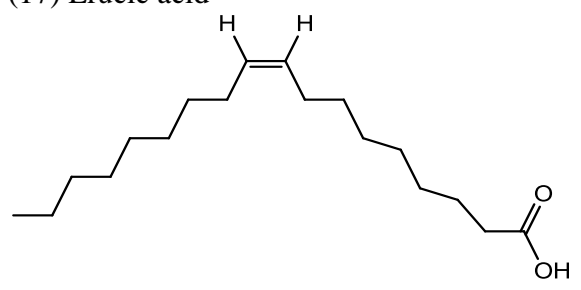

(19) Oleic acid

Fig. 2 Structures of fatty acids.

FAAH degrades the bioactive fatty acid amides like oleamide, endogenous cannabinoid, anandamide and myristic amide to their corresponding fatty acids.

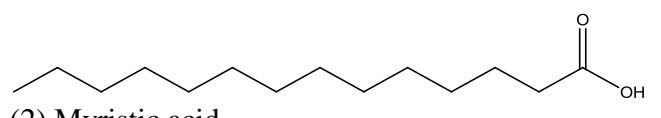

(2) Myristic acid

(4) Palmitic acid

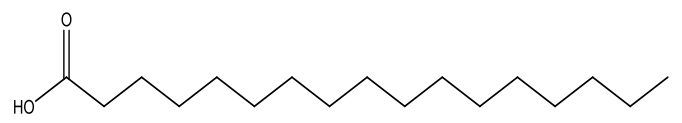

(6) Beptadecanoic acid<smiles>CCCCCCC(O)C/C=C/CCCCCCCC(=O)O</smiles>

(8) Ricinoleic acid<smiles>CC/C=C\C/C=C\C/C=C\CCCCCCCC(=O)O</smiles>

(10) $\alpha$-Linolenic acid

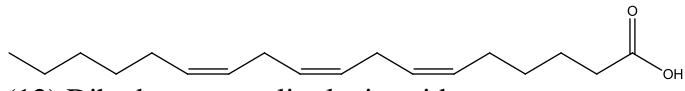

(12) Dihydro gamma linolenic acid

(13)

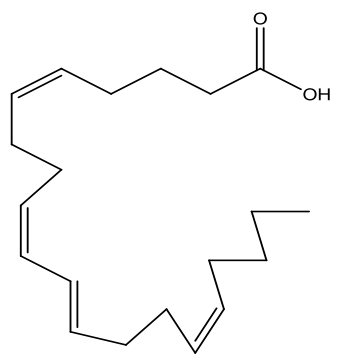

(14) Arachidonic acid

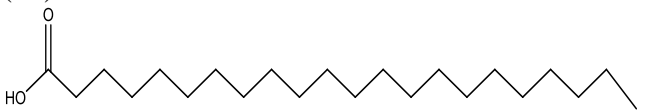

(16) Behenic acid

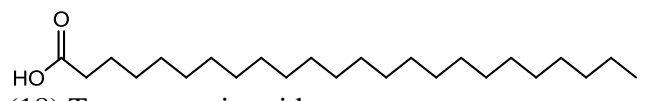

(18) Tetracosanoic acid

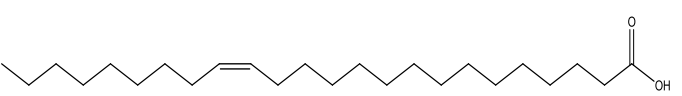

(20) Nervonic acid
Hence, these corresponding fatty acids are serving to terminate the signaling functions of these molecules. The polyunsaturated substrate anandamide was more 
preferentially hydrolyzed by FAAH when compared to that (hydrolysis by FAAH) of monounsaturated substrates. The $O$-aryl carbamates and $\alpha$-keto heterocytes inhibits the FAAH. As pain therapeutics [3], URB597, PF-622 and PF-750, relatively selective inhibitors of the enzyme FAAH (fatty acid amide hydrolase), are discovered recently as most potent and selective inhibition on FAAH target [4] because it regulates the pain relief mechanism (nociceptive mechanism). Hence, FAAH considered as promising target (Figs. 3-8).

Generally, the thought on property of URB597 is as follows: FAAH is the primary degradatory enzyme for anandamide of endocannabinoids. Then, the inhibition

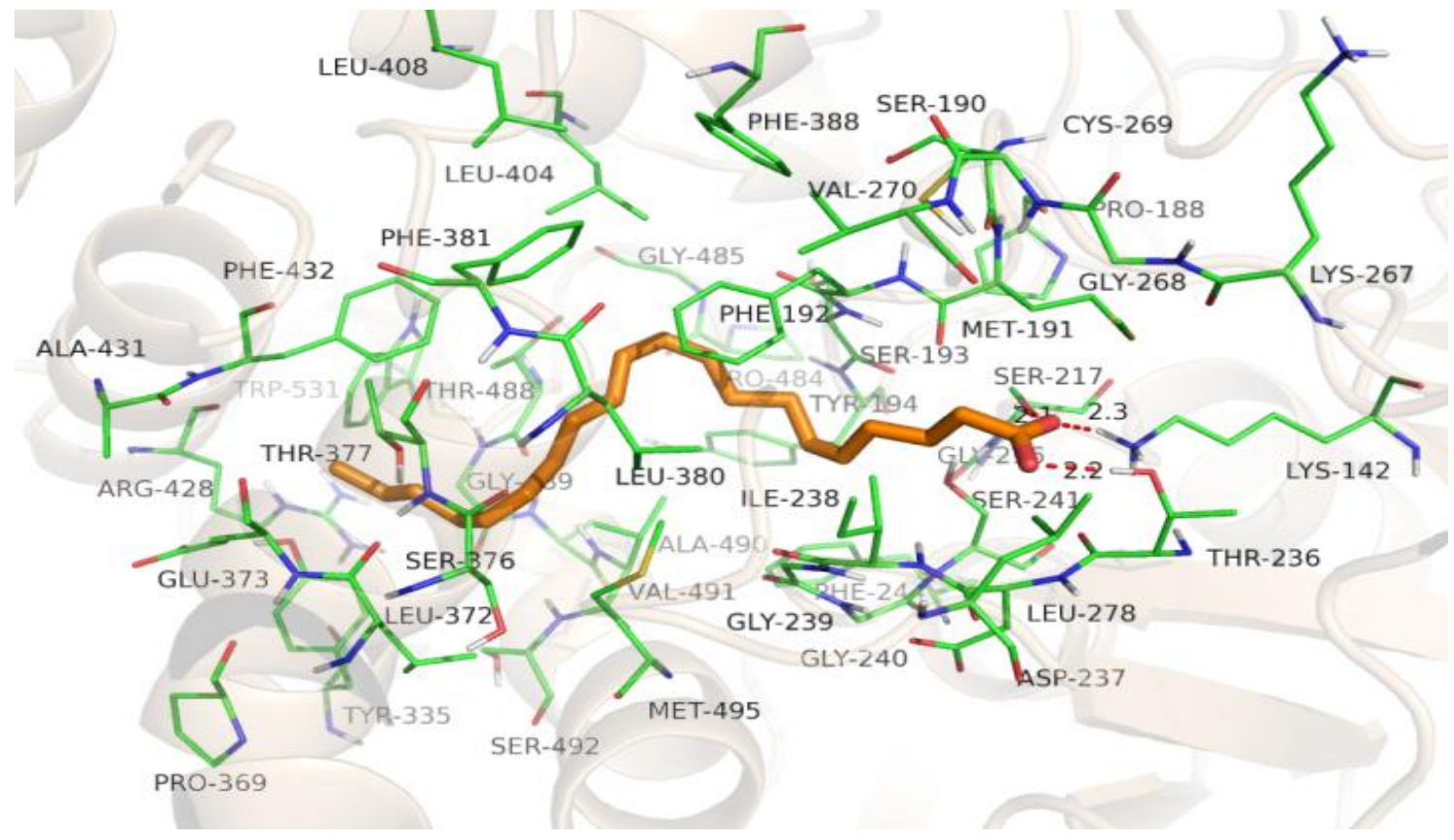

Fig. 3 3D structure of ADA.

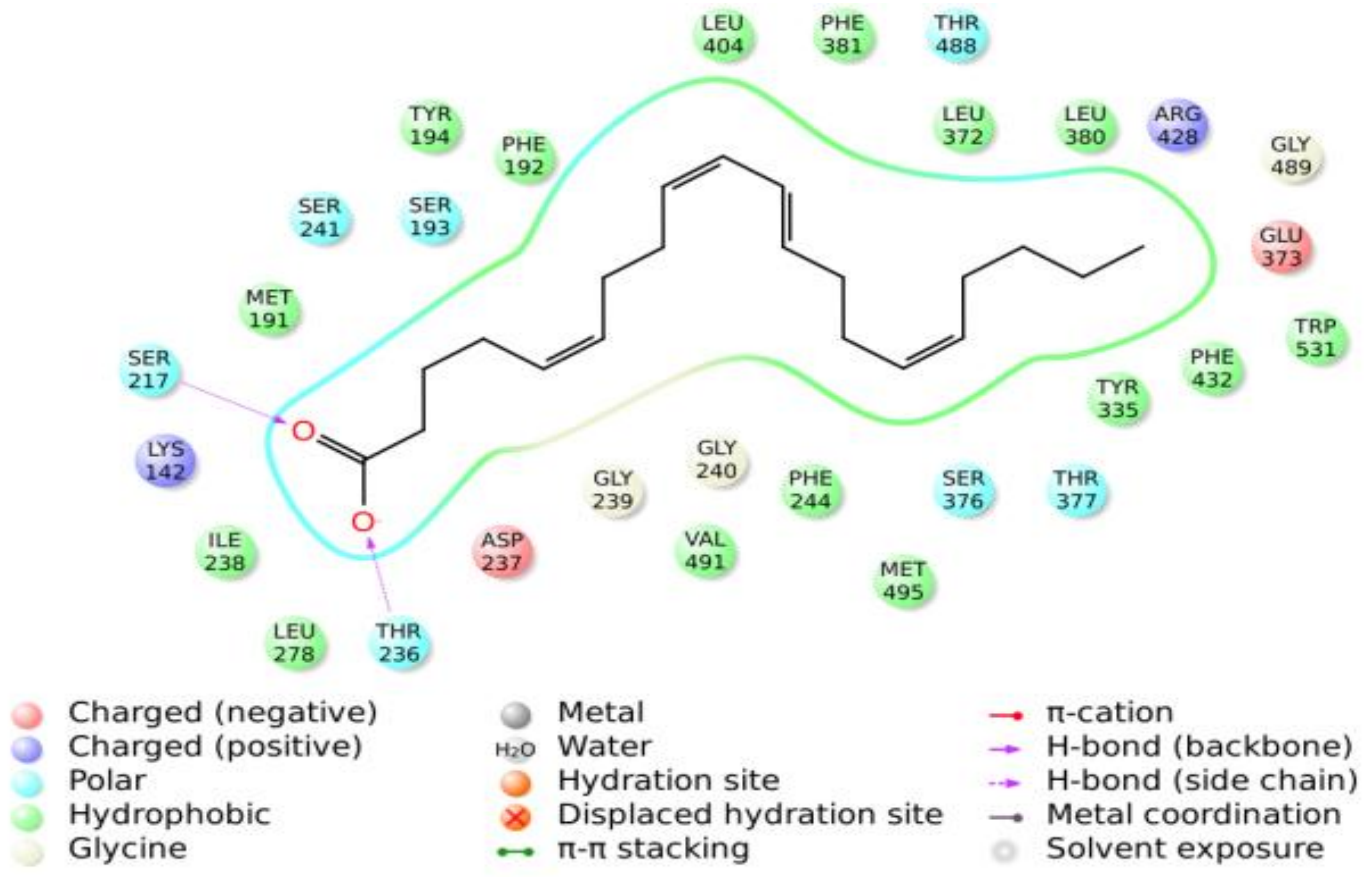

Fig. 4 2D structure of ADA. 
Comparative and Computer Assisted Drug Designing of Fatty Acids Isolated From Flowers, Leaves, $\quad 587$ Stem bark, Root bark and Nuts of Semecarpus anacardium L. f. (Anacardiaceae)

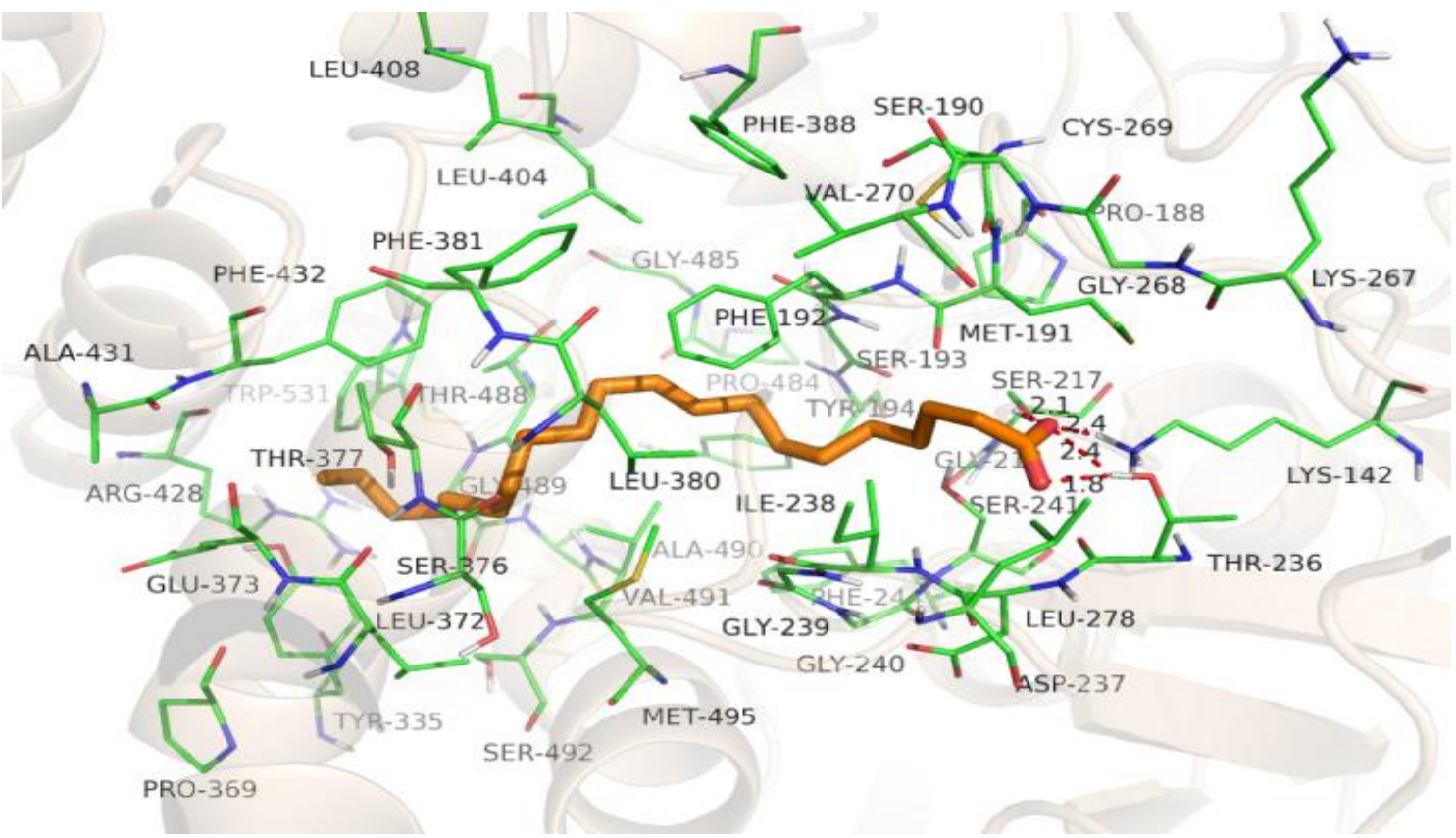

Fig. 5 3D structure of GA.

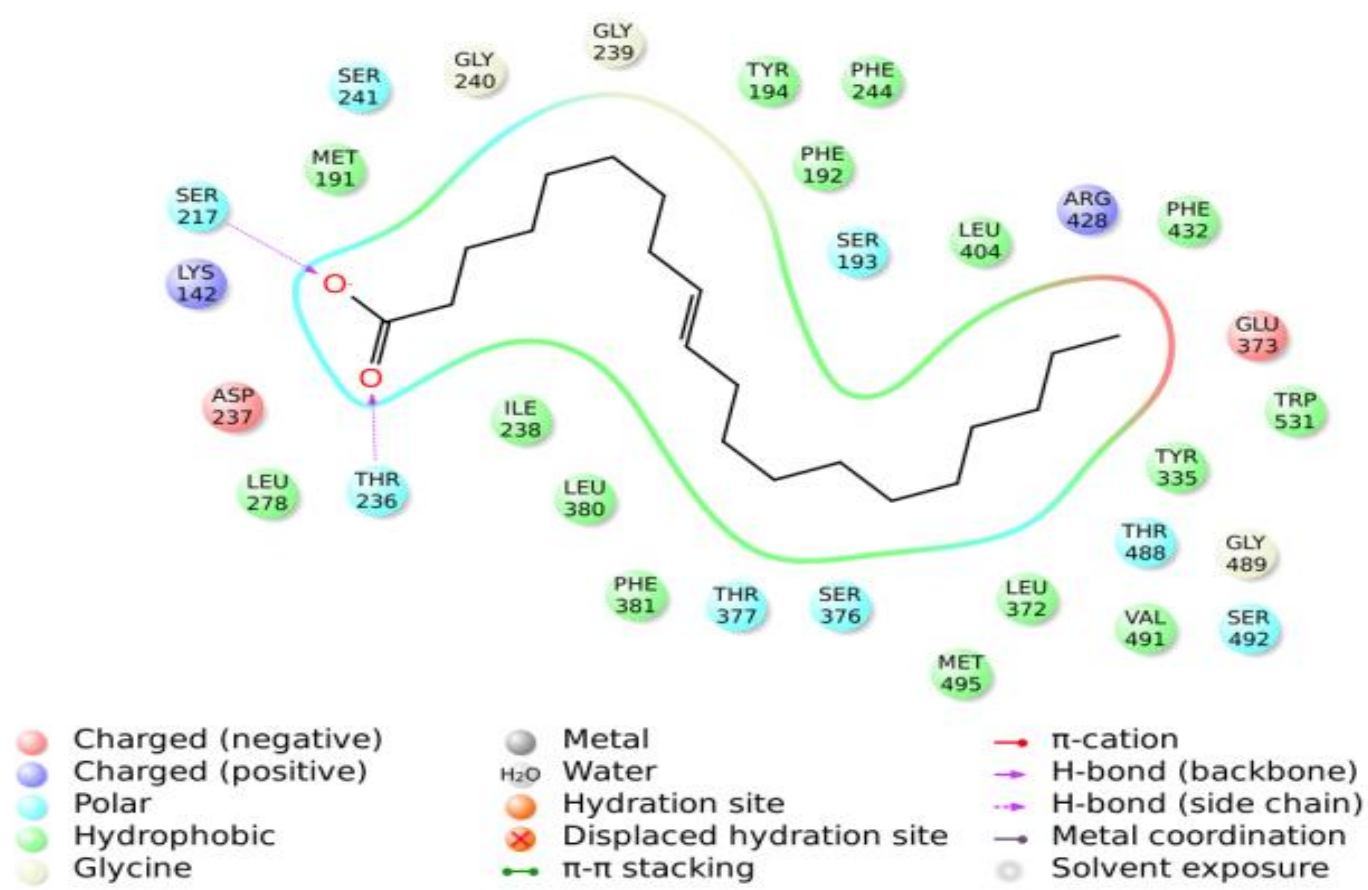

Fig. 6 2D structure of GA.

of FAAH leads to an accumulation of anandamide in both the CNS (central nervous system) and periphery. Subsequently, anandamide activates cannabinoid receptors. Therefore, URB597 can increase the production of endocannabinoids and show the antidepressant and analgesic effects.
2.5 Exploration of Probable Binding Modes of Fatty Acids, Using FAAH

\subsubsection{Dataset Preparation}

A dataset comprising of 20 fatty acids were drawn in ChemDraw and converted into 3D-molecules with all 


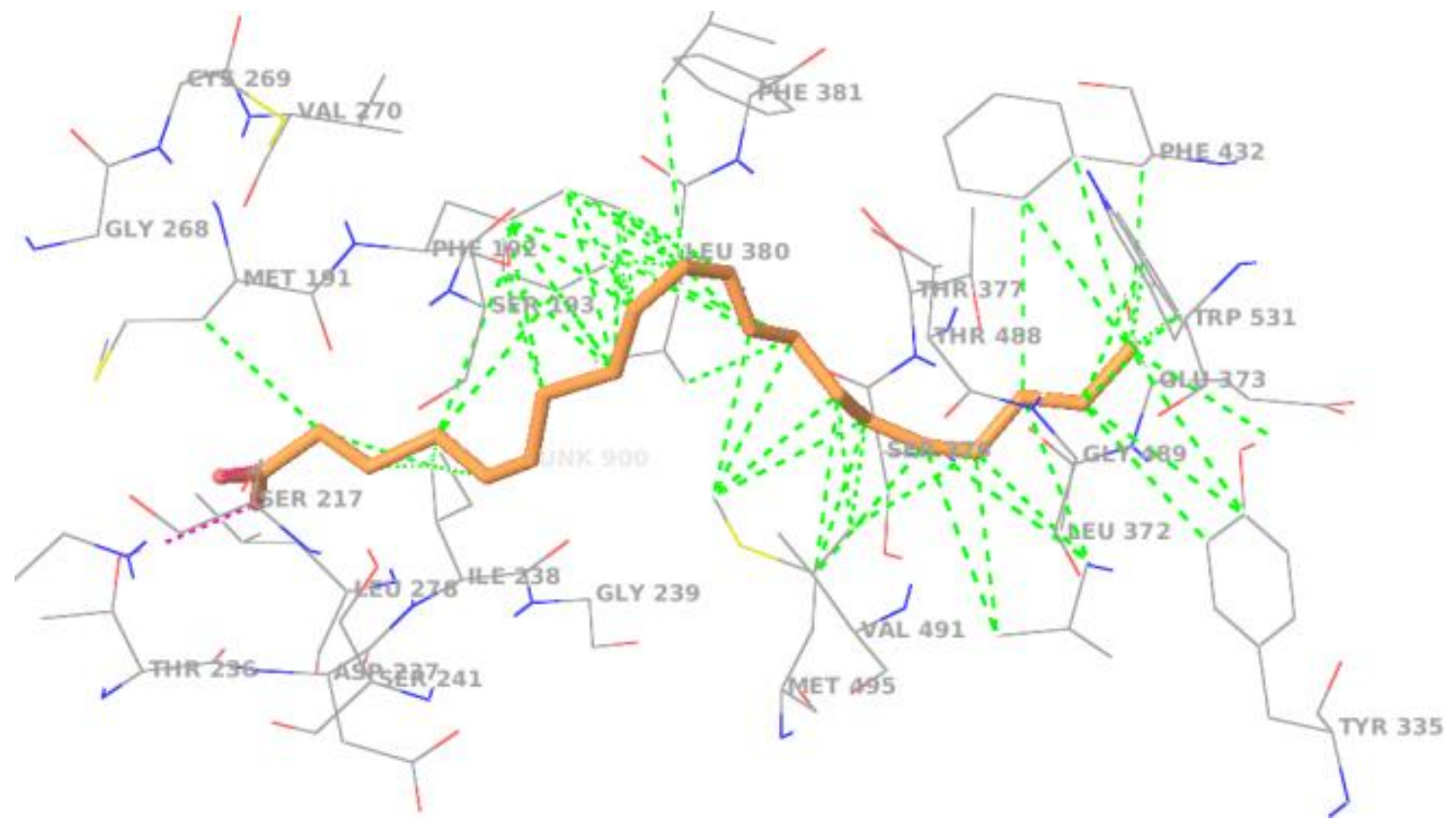

Fig. 7 ADA in the active site of FAAH.

Pink: hydrogen bond; green: hydrophobic interaction.

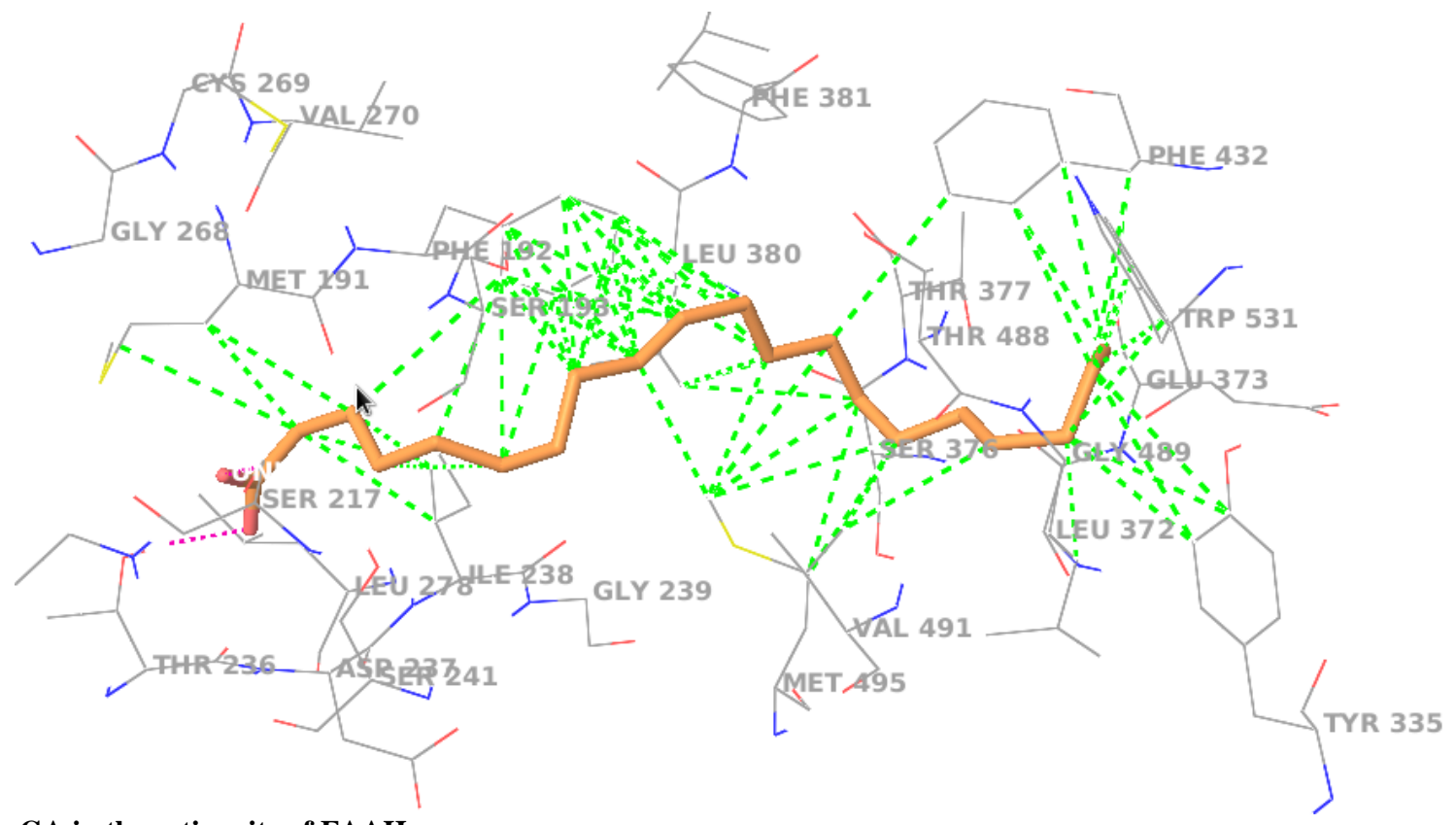

Fig. 8 GA in the active site of FAAH.

Pink: hydrogen bond; green: hydrophobic interaction.

possible tautomers and chiral centers. The converted 3D-molecules were minimized with OPLS-2005 force field using water as solvent in the GB/SA continuum solvation model [5] distance dependent electrostatic treatment was done at dielectric constant of 1.0; and the cutoff for potential energy calculation was kept extended. The extended cutoff includes contribution of van der Waals interaction (8.0), electrostatic (20), and hydrogen bond (4.0). The minimization of molecules was carried out using PRCG (Polak-Ribiere Conjugate Gradient) method with maximum of 5,000 iterations. The minimized compounds were used for protein 
Comparative and Computer Assisted Drug Designing of Fatty Acids Isolated From Flowers, Leaves, 589 Stem bark, Root bark and Nuts of Semecarpus anacardium L. f. (Anacardiaceae)

preparation.

\subsubsection{Protein preparation}

The crystal structure of human FAAH (PDB ID: 3K84) has been selected for the docking studies. Molecular docking study has been performed in order to explore the binding mode and understanding of key active site residues of the extracted natural compounds such as fatty acids in FAAH. The FAAH structures were prepared by adjusting bond orders, tautomers and adding hydrogen atoms using protein preparation wizard of Schrödinger software graphical user interface Maestro V9.3 [6]. Further, the proteins were minimized by OPLS_2005 force field with converge heavy atoms to RMSD $0.3 \AA$ relative to original protein structure.

\subsubsection{Docking Studies}

The docking study has been carried out on minimized 20 fatty acids with prepared FAAH proteins using Schrödinger docking program module [7]. The prepared protein structures $(3 \mathrm{~K} 84,1 \mathrm{~T} 49)$ were used for grid generation using the default value of protein atom scaling (1.0) within a cubic box centered on the co-crystal ligand. No constraints were imposed and extra precision (XP) docking of ligands was carried out with default value of ligand atom scaling (0.8). The post docking minimization has been carried out and maximum of 10 poses per ligand was saved. The obtained docked complex structures were analyzed and the compounds were prioritized by using docking score, interactions with active site residues.

\section{Results and Discussion}

20 fatty acids exist in flowers, leaves, stem barks, root barks and nuts of Semecarpus anacardium L. f..

Table 1 shows Existence of isolated 20 fatty acids in nuts (i.e., LA, MA, PDA, PL, PALA, HAD, SA, RA,
LA, ALA, GLA, DGL, AA, ADA, GA, BA, EA, TCA, OA \& NA), where 5 fatty acids exist in flowers (i.e., PL, SA, BA, TCA \& NA), 3 fatty acids in leaves (i.e., PL, SA \& OA), 5 fatty acids in stem bark (i.e., PL, SA, LA, TCA \& OA) and 8 fatty acids in root bark (i.e., PL, SA, LA, AA, BA, TCA, OA \& NA) of Semecarpus anacardium L. f., which were shown in Fig.2.

\subsection{Molecular Docking}

Molecular docking study has been performed in order to explore the binding mode and understanding of key active site residues of the extracted natural compounds such as fatty acids in FAAH. The probable binding modes of best docked compounds are shown in Figs. 3-8 and its interaction profile is shown in Table 2. In Table 3, the docking parameters and physicochemical properties, fatty acids in FAAH targets are shown clearly.

\subsection{ADA in the Active Site of FAAH}

The carboxylic two oxygen atoms showed hydrogen bond interactions with Thr236, Ser217 residues. The long aliphatic chain of the ligand showed hydrophobic interactions with Met191, Phe192, Met495, Leu380, Val491, Leu372, Tyr335, Trp531 and Phe432 (Figs. 3 and 4).

\subsection{GA in the Active Site of FAAH}

The carboxylic two oxygen atoms i.e., oxygen in hydroxyl group showed hydrogen bond interactions with Thr236, and oxygen in carbonyl group showed hydrogen bond interactions with Ser217 residues. The long aliphatic chain oriented towards hydrophobic interactions include: Met191, Phe192, Met495, Leu380, Val491, Leu372, Tyr335, Trp531 and Phe432 (Figs. 5 and 6).

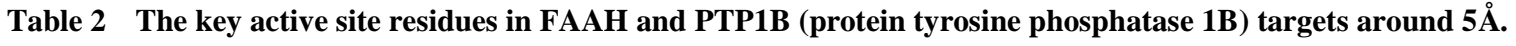

\begin{tabular}{ll}
\hline Target & Active site (5尺) \\
\hline & LYS142, MET191, PHE192, SER193, TYR194, SER217, THR236, ASP237, ILE238, GLY239, GLY240, SER241, \\
& PHE244, LEU278, TYR335, LEU372, GLU373, SER376, THR377, LEU380, ARG428, PHE432, THR488, GLY489, \\
FAAH & VAL491, SER492, MET495, TRP531, LYS142, MET191, PHE192, SER193, TYR194, SER217, THR236, ASP237, \\
& ILE238, GLY239, GLY240, SER241, PHE244, LEU278, TYR335, LEU372, GLU373, SER376, THR377, LEU380, \\
& ARG428, PHE432, THR488, GLY489, VAL491, SER492, MET495 and TRP531 \\
\hline
\end{tabular}


Table 3 The docking parameters and physicochemical properties of fatty acids in FAAH target.

\begin{tabular}{|c|c|c|c|c|c|c|c|c|c|}
\hline S. No & ID & gscore & evdw & ecoul & energy & emodel & Mol.wt & $\log \mathrm{P}$ & PSA \\
\hline 14 & $\mathrm{ADA}$ & -10.27 & -39.22 & -5.34 & -44.56 & -57.21 & 304.47 & 6.38 & 50.24 \\
\hline 15 & GA & -9.96 & -38.8 & -7.56 & -46.36 & -56.58 & 310.52 & 6.55 & 47.95 \\
\hline 20 & NA & -9.66 & -37.44 & -5.95 & -43.39 & -51.71 & 366.63 & 7.47 & 49.91 \\
\hline 8 & RA & -9.45 & -36.66 & -7.7 & -44.36 & -60.09 & 298.47 & 4.5 & 71.21 \\
\hline 13 & AA & -8.66 & -36.33 & -9.11 & -45.44 & -43.37 & 312.54 & 6.6 & 47.74 \\
\hline 9 & LA & -8.34 & -30.05 & -6.17 & -36.22 & -47.68 & 280.45 & 5.5 & 50.79 \\
\hline 5 & PALA & -7.75 & -31.85 & -5.32 & -37.17 & -38.36 & 254.41 & 4.89 & 50.61 \\
\hline 7 & SA & -7.34 & -35.39 & -6.39 & -41.78 & -56.09 & 284.48 & 5.9 & 50.38 \\
\hline 19 & OA & -7.27 & -36.86 & -5.91 & -42.77 & -56.01 & 282.47 & 5.74 & 50.73 \\
\hline 4 & PL & -7.26 & -32.06 & -5.14 & -37.2 & -47.58 & 256.43 & 5.08 & 50.59 \\
\hline 6 & HDA & -7.26 & -34.45 & -6.89 & -41.33 & -49.9 & 270.45 & 5.52 & 50.08 \\
\hline 12 & DGL & -7.08 & -28.17 & -9.35 & -37.51 & -47.84 & 278.43 & 5.53 & 50.57 \\
\hline 10 & ALA & -6.77 & -33.87 & -6.16 & -40.03 & -50.21 & 278.43 & 5.47 & 50.1 \\
\hline 2 & MA & -6.73 & -21.9 & -9.75 & -31.65 & -33.07 & 228.37 & 4.45 & 47.95 \\
\hline 17 & EA & -5.63 & -39.4 & -6.87 & -46.27 & -57.72 & 338.57 & 7.3 & 50.03 \\
\hline 11 & GLA & -5.54 & -26.92 & -9.82 & -36.75 & -45.96 & 250.38 & 4.89 & 50.36 \\
\hline 1 & LA & -4.81 & -24.59 & -5.9 & -30.49 & -38.31 & 200.32 & 3.69 & 50.27 \\
\hline 18 & TCA & -4.64 & -41.73 & -4.53 & -46.26 & -65.56 & 368.64 & 7.99 & 44.64 \\
\hline 3 & PDA & -4.6 & -27.57 & -9.39 & -36.96 & -35.36 & 242.4 & 4.81 & 50.3 \\
\hline 16 & BA & -4.37 & -40.06 & -4.84 & -44.9 & -60.06 & 340.59 & 7.43 & 48.73 \\
\hline
\end{tabular}

3.4 The Hydrogen Bond and Hydrophobic Interactions

The hydrogen bond and hydrophobic interactions of FAAH with ADA was shown in Fig. 7.

The hydrogen bond and hydrophobic interactions of FAAH with GA was shown in Fig. 8.

\section{Conclusions}

The isolated 20 fatty acids from nuts, 8 fatty acids from root bark, 3 fatty acids from leaves, 5 fatty acids from stem bark and 5 fatty acids flowers of Semecarpus $\operatorname{anacardium~L.~f.,~were~elaborated~in~Table~} 1$ and each structural elucidation was confirmed individually in our laboratory [8-10]. To explore the binding mode and understanding of key active site residues of the extracted natural compounds, such as fatty acids in the active site, docking studies were carried out by taking the crystal structure of human FAAH (PDB ID: 3K84). Docking studies indicated the presence of LYS142, MET191, PHE192, SER193, TYR194, SER217, THR236, ASP237, ILE238, GLY239, GLY240, SER241， PHE244，LEU278, TYR335, LEU372, GLU373, SER376, THR377, LEU380, ARG428,
PHE432，THR488， GLY489，VAL491，SER492, MET495, TRP531, LYS142, MET191, PHE192, SER193，TYR194， SER217， THR236，ASP237, ILE238, GLY239, GLY240, SER241, PHE244, LEU278，TYR335，LEU372， GLU373， SER376, THR377, LEU380, ARG428, PHE432, THR488, GLY489, VAL491, SER492, MET495 and TRP531 amino acids in the active site. The carboxylic two oxygen atoms i.e., oxygen in hydroxyl group showed hydrogen bond interactions Thr236, and oxygen in carbonyl group showed hydrogen bond interactions with Ser217 residues. The long aliphatic chain of the ligand showed hydrophobic interactions with Met191, Phe192, Met495, Leu380, Val491, Leu372, Tyr335, Trp531 and Phe432. Hence the molecular docking studies could provide substantial design clues for the development of novel, potent inhibitors for FAAH targets.

\section{References}

[1] Madhavachetty, K., Sivaji, K. K., and Rao, K. T. 2008. "Flowering Plants of Chittor District", A. P. India. 76: 131. 
[2] Murthy, S. S. N. 1992. "New Biflavonoid from Semecarpus anacardium Linn." Clin. Acta Turcica. 20: 33-7.

[3] Wei, B. Q., Mikkelsen, T. S., McKinney, M. K., Lander E. S., and Cravatt, B. F. 2006. "A Second Fatty Acid Amide Hydrolase with Variable Distribution among Placental Mammals." J. Biol. Chem. 281: 36569-78.

[4] Ahn, K., Johnson, D. S., Fitzgerald, L. R., Liimatta, M., Arendse, A., Stevenson, T., Lund, E. T, Nugent, R. A., Nomanbhoy, T. K., Alexander, J. P., and Cravatt, B. F. 2007. "Novel Mechanistic Class of Fatty Acid Amide Hydrolase Inhibitors with Remarkable Selectivity", Biochemistry 46 (45): 13019-30.

[5] Still, W. C., Tempczyk, A., Hawley, R. C., and Hendrickson, T. 1990. "Semi-analytical Treatment of Solvation for Molecular Mechanics and Dynamics." $J$. Am. Chem. Soc. 112 (16): 6127-9.

[6] Maestro, version 9.3, Glide version 2.8, Schrödinger, L.L.C., 2013. New York, NY.

[7] Friesner, R. A., Banks, J. L., Murphy, R. B., Halgren, T. A., Klicic, J. J., Mainz, D. T., Repasky, M. P., Knoll, E. H., Shelley, M., Perry, J. K., Shaw, D. E., Francis, P., and Shenkin, P. S. 2004. "Glide: A New Approach for Rapid,
Accurate Docking and Scoring. 1. Method and Assessment of Docking Accuracy." J. Med. Chem. 47 (7): 1739-49.

[8] Padmavathi, V., and Rao, B. K. 2013. "FT-IR Studies in Designing and Discovering the Potent Molecules as Drugs from Semecarpus anacardium linn. (Anacardiaceae family)", Venue, National Conference on Drug Designing and Discovery (DDD-2013).

[9] Padmavathi, V., and Rao, B. K. 2014. "Phytochemical and Antimicrobial, Anticancer Investigation of Semecarpus anacardium L.f., Flowers \& Leaves (Anacardiaceae family)", Venue, Abstract-Poster-171. In International Symposium on Nature Inspired Initiatives in Chemical Trends (NIICT-2014), March 2-5, CSIR-IICT, Hyderabad, India.

[10] Padmavathi, V, and Rao, B. K. 2014. "New Dimensions in Isolation, Anti-Inflammatory, Antimicrobial, Multi-metal Analysis of Semecarpus anacardium L.f., Stem Bark.” In Proceedings of International Conference on New Dimensions in Chemistry and Chemical Technologies Applications in Pharma Industry (NDCT-2014), JNTU-Hyderabad, June 23-25, 422-7. 